\title{
The cytoskeletal protein ezrin regulates EC proliferation and angiogenesis via TNF- $\alpha-$ induced transcriptional repression of cyclin A
}

\author{
Raj Kishore, Gangjian Qin, Corinne Luedemann, Evelyn Bord, Allison Hanley, \\ Marcy Silver, Mary Gavin, David Goukassain, and Douglas W. Losordo
}

Division of Cardiovascular Research, St. Elizabeth's Medical Center, Tufts University School of Medicine, Boston, Massachusetts, USA.

\begin{abstract}
TNF- $\alpha$ modulates EC proliferation and thereby plays a central role in new blood vessel formation in physiologic and pathologic circumstances. TNF- $\alpha$ is known to downregulate cyclin A, a key cell cycle regulatory protein, but little else is known about how TNF- $\alpha$ modulates EC cell cycle and angiogenesis. Using primary ECs, we show that ezrin, previously considered to act primarily as a cytoskeletal protein and in cytoplasmic signaling, is a TNF- $\alpha$-induced transcriptional repressor. TNF- $\alpha$ exposure leads to Rho kinase-mediated phosphorylation of ezrin, which translocates to the nucleus and binds to cell cycle homology region repressor elements within the cyclin A promoter. Overexpression of dominant-negative ezrin blocks TNF- $\alpha$-induced modulation of ezrin function and rescues cyclin A expression and EC proliferation. In vivo, blockade of ezrin leads to enhanced transplanted EC proliferation and angiogenesis in a mouse hind limb ischemia model. These observations suggest that TNF- $\alpha$ regulates angiogenesis via Rho kinase induction of a transcriptional repressor function of the cytoskeletal protein ezrin and that ezrin may represent a suitable therapeutic target for processes dependent on EC proliferation.
\end{abstract}

\section{Introduction}

Regulation of EC proliferation plays a key role in angiogenesis and vascular pathology $(1,2)$. Under both circumstances, TNF- $\alpha$ has been implicated as an important regulatory factor.

In ECs, TNF- $\alpha$ activates inflammatory responses and influences angiogenesis but can also negatively affect EC proliferation and induce EC apoptosis (3-6). In turn, the inhibition of EC proliferation and enhanced apoptosis in response to TNF- $\alpha$ have significant bearing on endothelial recovery following balloon angioplasty, which may contribute to the development of restenosis, the recurrent obstruction of coronary arteries that complicates a significant percentage of angioplasties. Indeed, we have previously reported that blockade of locally expressed TNF- $\alpha$ at the site of balloon injury improves the process of re-endothelialization (7). We and others have also reported that in vitro exposure of primary ECs to TNF- $\alpha$ inhibits their proliferation and impairs their ability to progress through the cell cycle $(5,6,8)$. This inhibitory effect of TNF- $\alpha$, at least partially, depends on the downregulation of cell cycle regulatory genes, including that of cyclin A $(5,6)$. Recently we have reported that at the molecular level the inhibitory effect of TNF- $\alpha$ on cyclin A transcription is partly mediated by the recruitment of an approximately $80-\mathrm{kDa}$ protein that binds specifically to the cell cycle homology region (CHR) transcriptional corepressor cis-elements in the promoter of cyclin A (9).

Here we demonstrate that this CHR-binding protein is the cytoskeleton protein ezrin, which in response to TNF- $\alpha$ translocates to the nucleus and functions as a transcriptional repressor. Ezrin

Nonstandard abbreviations used: BAEC, bovine arterial EC; ChIP, chromatin immunoprecipitation; CHR, cell cycle homology region; DN ezrin, dominant-negative ezrin; EMSA, electrophoretic mobility shift assay; ERM, ezrin, radixin, and moesin; HUVEC, human umbilical vein EC; IB4, isolectin B4.

Conflict of interest: The authors have declared that no conflict of interest exists.

Citation for this article: J. Clin. Invest. 115:1785-1796 (2005).

doi:10.1172/JCI22849. is a member of the ezrin, radixin, and moesin (ERM) subfamily of cytoskeletal proteins and bears significant homology with other cytoskeletal proteins (10-12). ERM proteins play a crucial role in the formation of microvilli, cell-cell adhesion, maintenance of cell shape, cell motility, and membrane trafficking (13-15). Although initially believed to function solely as cytoskeleton "organizers," recent evidence has shown that ERM proteins, including ezrin, are not only involved in the cytoskeletal organization but are also involved in a number of signaling pathways (16). These findings led to the identification of other functional properties of ezrin including cellular proliferation and apoptosis and their modulation by various stimuli in a cell type-specific manner $(16,17)$. However, none of these studies has yet demonstrated the involvement of ezrin in the regulation of gene transcription and cell cycle inhibition. In the present study we provide evidence that TNF- $\alpha$ treatment of ECs not only elevates ezrin expression but also initiates a RhoA signaling cascade leading to ezrin phosphorylation, nuclear translocation, and inhibition of cyclin A transcription via direct transcriptional repressor activity.

\section{Results}

Ezrin binds to cyclin A CHR elements. We have previously shown that exposure of ECs to TNF- $\alpha$ downregulates cyclin A transcription via recruitment of an $80-\mathrm{kDa}$ protein, which binds specifically to CHR corepressor elements in the cyclin A promoter (9). Partial purification and matrix-assisted laser desorption ionization (MALDI) analysis identified this protein as ezrin, a member of the cytoskeletal ERM subfamily of proteins. This was an intriguing finding, since ezrin is not known to bind to promoter cis-elements and regulate transcription of any known gene. To substantiate this finding, we first investigated the ability of ezrin to bind cyclin A CHR elements. Nuclear extracts from control and TNF- $\alpha$-treated ECs were mixed with biotin-labeled CHR oligonucleotides, and the bound proteins were captured on avidin-agarose beads. Eluted 

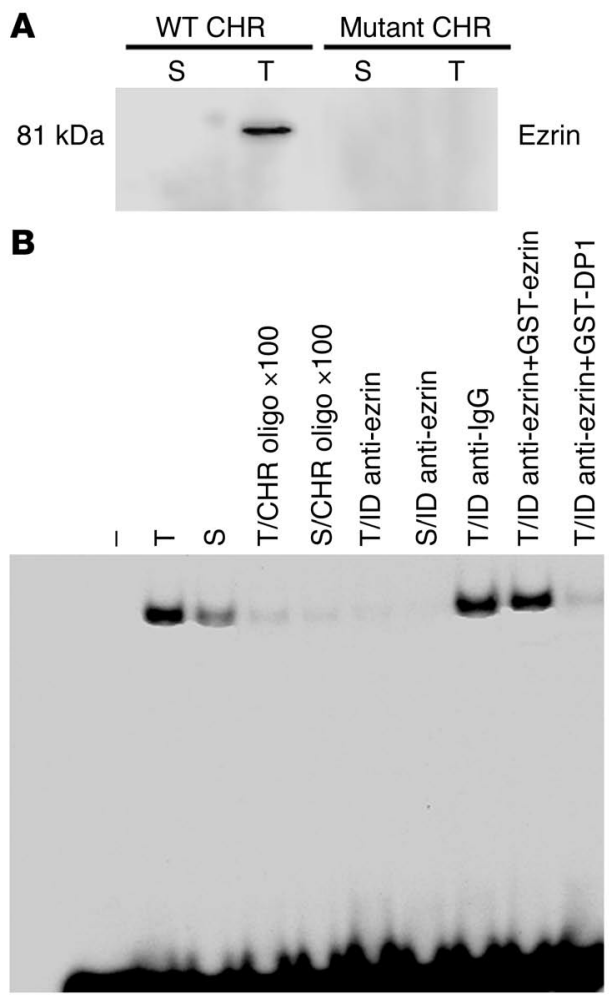

C

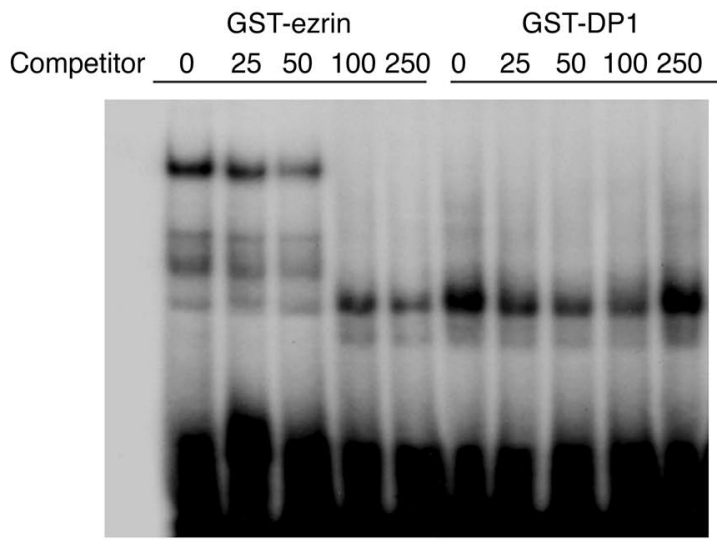

Figure 1

Ezrin is a TNF- $\alpha$-inducible cyclin A CHR-binding protein. (A) Nuclear extracts from serum-stimulated (S) and serum-stimulated and TNF- $\alpha$-treated (T) BAECs were incubated with biotinylated WT or mutant CHR oligonucleotides. Protein-DNA complexes were captured on avidin-agarose columns. Eluted proteins from columns were analyzed for ezrin protein expression in Western blots. (B) Nuclear extracts from serum-treated or TNF- $\alpha-$ treated cells were immunodepleted (ID) of ezrin by anti-ezrin antibodies. Depleted extracts alone or depleted extracts from TNF- $\alpha$-treated cells reconstituted with recombinant-ezrin protein (GST-ezrin) or control DP1 protein (GST-DP1) were then analyzed for CHR-binding activity. $\times 100,100$-fold molar excess of competing unlabeled oligonucleotide. (C) GST-ezrin or GST-DP1 protein (1 $\mu \mathrm{g})$ was incubated with radiolabeled oligonucleotides spanning $\mathrm{CHR}$-binding elements in EMSA experiments. For competition experiments, the indicated molar concentration of unlabeled oligonucleotides was added to the reaction mix before the addition of radiolabeled probe. Autoradiographs shown are representative of at least 4 similar experiments.

proteins from beads were subjected to Western blot analysis for ezrin. As shown in Figure 1A, CHR oligonucleotide-nuclear protein complex revealed specific binding of ezrin to the CHR oligonucleotides in the nuclear extracts of TNF- $\alpha$-treated ECs. This CHR-ezrin interaction was specific, since control oligonucleotides with mutations in the $\mathrm{CHR}$ region (mutant $\mathrm{CHR}$ ) failed to capture ezrin from TNF- $\alpha$-treated nuclear extracts. That TNF- $\alpha$ induced ezrin-CHR binding activity was further evident from ezrin immunodepletion experiments. Endogenous ezrin was immunodepleted from control and TNF- $\alpha$-treated nuclear extracts by treatment of extracts with anti-ezrin antibodies over 5 rounds. Immunodepleted extracts were then analyzed by electrophoretic mobility shift assay (EMSA) for CHR-binding activity. As shown in Figure 1B, depletion of ezrin from TNF- $\alpha$-treated nuclear extracts completely abrogated TNF- $\alpha$-induced upregulation of CHR-binding activity observed in similarly treated but nondepleted nuclear extracts or extracts treated with nonspecific antibody. Addition of recombinant ezrin back to the depleted TNF- $\alpha$ extract reconstituted CHR-binding activity. The specificity of CHR-ezrin binding was further confirmed by EMSA assays using recombinant-ezrin protein (GST-ezrin). Recombinant ezrin formed complexes with CHR oligonucleotides, which were effectively competed with increasing excess molar concentrations of unlabeled oligonucleotides indi- cating the specificity of the binding activity (Figure 1C). A control recombinant-DP1 protein did not form a complex of similar mobility with CHR oligonucleotides, which indicates the specificity of ezrin-CHR binding.

TNF- $\alpha$ modulates ezrin expression and subcellular distribution. No prior study to our knowledge has documented nuclear expression and/or stimulus-induced nuclear translocation of full-length ezrin. Since nuclear translocation would be required in order for ezrin to bind cyclin A CHR elements, we examined the subcellular distribution of ezrin in control and TNF- $\alpha$-treated ECs using a variety of experimental strategies. Immunocytochemical staining (data not shown) of control and TNF- $\alpha$-treated cells revealed a similar shift of ezrin from cytoplasmic/perinuclear in control cells to predominantly nuclear in TNF- $\alpha$-treated cells. This observation was further corroborated by laser confocal microscopy, which revealed that upon TNF- $\alpha$ stimulation ezrin translocates to the nucleus and colocalizes with propidium iodide-stained nuclei (Figure 2A). TNF- $\alpha$ treatment of ECs for 24 hours not only upregulated ezrin expression in whole-cell lysates but also substantially shifted the localization of ezrin predominantly to the nuclear compartment as opposed to the predominantly cytoplasmic localization in the control cells (Figure 2B). Interestingly, expression and localization of radixin and moesin, 2 related proteins, did not change in 
A

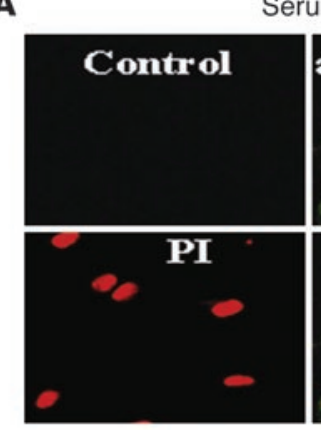

B

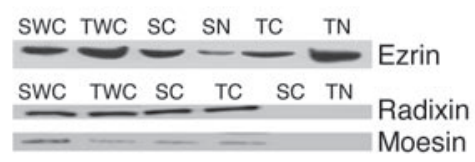

TNF

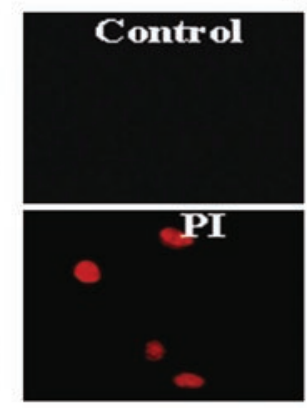

c
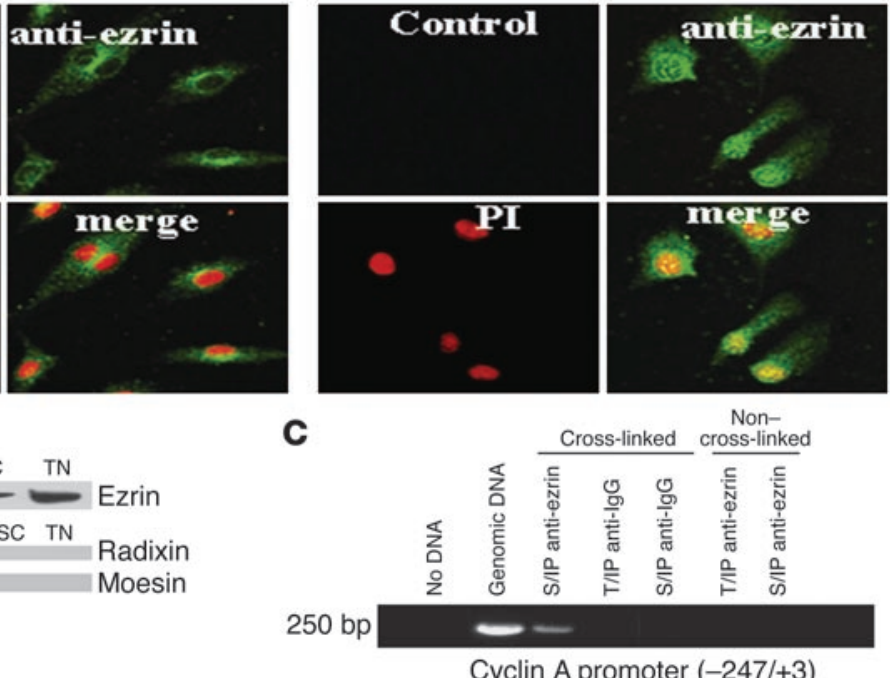

Figure 2

TNF- $\alpha$ translocates ezrin to the nucleus. (A) Representative laser confocal microscopy images showing nuclear translocation and colocalization of ezrin (green stain) with nuclei stained with propidium iodide (PI; red stain) in TNF- $\alpha$-treated cells (yellow in the merged images). (B) Whole-cell (WC), cytoplasmic (C), and nuclear $(\mathrm{N})$ extracts from BAECs stimulated with serum alone or treated with TNF- $\alpha$ were analyzed for ERM protein expression by Western blots. (C) Representative PCR gel photograph showing amplification of $250 \mathrm{bp}$ of the cyclin A promoter encompassing $\mathrm{CHR}$ elements, using DNA from anti-ezrin antibody chromatin immunoprecipitates from serum-treated or TNF- $\alpha$-treated cells. Isotype-matched rabbit anti-lgG antibody was used as a control for ChIP. response to TNF- $\alpha$ (Figure 2B). Furthermore, TNF- $\alpha$-induced ezrin translocation appears to be cell type specific, since treatment of E19P rat VSMCs did not induce nuclear ezrin expression (see Supplemental Figure 1; supplemental material available online with this article; doi:10.1172/JCI22849DS1).

Nuclear expression and TNF- $\alpha$ modulation of ezrin was confirmed by the binding of ezrin to cyclin A CHR elements in vivo, in chromatin immunoprecipitation (ChIP) experiments. As shown in Figure 2C, PCR amplification of DNA isolated from anti-ezrinimmunoprecipitated chromatin-protein complexes from TNF- $\alpha$ treated ECs specifically detected 250 bp of the cyclin A promoter, while that of DNA isolated from anti-DP1 immunoprecipitates did not; this further corroborates ezrin-CHR interaction. Taken together, these data demonstrate that TNF- $\alpha$ modulates the subcellular distribution of ezrin, translocating it to the nucleus, where it binds the target transcriptional repressor CHR elements of the cyclin A promoter. To further substantiate that TNF- $\alpha$ treatment of ECs translocates full-length ezrin to the nucleus, we transfected cells with ezrin vectors expressing either full-length WT ezrin or a truncated N-terminal region (phosphorylation-defective, dominant-negative isoform; DN ezrin), both tagged with VSVG protein. ECs were transiently transfected with the WT or DN ezrin expression vector, and the efficacy of transgene expression and TNF- $\alpha$-induced nuclear translocation was determined by VSVG-tag immunostaining and confocal microscopy. As shown in Supplemental Figure 2A, VSVG protein expression was identical in WT ezrin- and DN ezrin-transfected cells. Interestingly, Western blot analysis revealed that TNF- $\alpha$ treatment of DN ezrin-transfected cells failed to translocate DN ezrin in the nuclear compartment. Confocal microscopy of anti-VSVG antibody-stained ECs revealed that ectopically expressed WT ezrin was found exclusively in the cytoplasm of control cells, while TNF- $\alpha$ treatment led to the translocation of ezrin (i.e., the VSVG tag) to the nucleus (Supplemental Figure 2B). These data further corroborate ezrin nuclear translocation in response to TNF- $\alpha$. Furthermore, TNF- $\alpha-$ induced ezrin translocation appears to be dependent on TNF- $\alpha$ dose. Ezrin immunostaining by confocal microscopy in ECs treated with increasing doses of TNF- $\alpha$ revealed that ezrin nuclear translocation began to appear at a dose of $5 \mathrm{ng} / \mathrm{ml}$ and became predominantly evident at a dose of $20 \mathrm{ng} / \mathrm{ml}$ (Supplemental Figure 3).
Dominant-negative ezrin rescues ECs from $T N F-\alpha$-mediated inhibition in proliferation. Having documented TNF- $\alpha$-induced nuclear translocation and binding to cyclin A CHR elements, we next sought to determine the functional consequences of TNF- $\alpha$-mediated ezrin modulation in ECs. To delineate the role of ezrin in cyclin A transcription and EC proliferation in response to TNF- $\alpha$, we used WT ezrin and $\mathrm{DN}$ ezrin expression vectors in transient transfection experiments. To rule out that overexpression of DN ezrin might compromise cellular architecture, we examined the effect of both WT ezrin and DN ezrin overexpression on F-actin organization. Compared with untransfected cells (Figure 3A), overexpression of either WT or DN ezrin had no apparent effect on EC cytoskeleton, as evident by phalloidin staining of F-actin (Figure 3, C and E). TNF- $\alpha$ treatment of control or WT ezrin-transfected cells led to an increase in the condensation of actin stress fiber formation, which was markedly inhibited in DN ezrin-transfected, TNF- $\alpha$-treated cells (Figure 3, D and F). Similar changes in the actin reorganization of bovine arterial ECs (BAECs) in response to TNF- $\alpha$ have been described by others $(18,19)$. To assess the effect of ezrin modulation by TNF- $\alpha$ on EC proliferation, transfected cells were treated or not treated with TNF- $\alpha$ and BrdU $(50 \mu \mathrm{g} / \mathrm{ml})$. As shown in Figure 3G, in mock-transfected cells (empty vector), TNF- $\alpha$ substantially reduced EC proliferation, as assessed by BrdU incorporation (BrdU-positive cells, $216 \pm 2.3 / \mathrm{mm}^{2}$ in control vs. $58 \pm 3.1 / \mathrm{mm}^{2}$ in TNF- $\alpha$-treated cells, $\left.P<0.01\right)$. Overexpression of WT ezrin reduced proliferation by itself (BrdU-positive cells, $\left.163 \pm 3.2 / \mathrm{mm}^{2}, P<0.05\right)$ and exacerbated the effect of TNF- $\alpha$ (BrdU-positive cells, $11 \pm 1.6 / \mathrm{mm}^{2}$ vs. $58 \pm 3.1 / \mathrm{mm}^{2}$ in empty vectortransfected cells, $P<0.01)$. Most importantly, overexpression of DN ezrin attenuated the repressive effect of TNF- $\alpha$ on EC proliferation (BrdU-positive cells, $117 \pm 3.4 / \mathrm{mm}^{2}$ in DN ezrin-transfected cells vs. $11 \pm 1.6 / \mathrm{mm}^{2}$ in WT ezrin-transfected cells, $\left.P<0.001\right)$. These data provided evidence that ezrin mediates TNF- $\alpha$-induced growth arrest in ECs and that blockade of ezrin function could alter EC function.

Dominant-negative ezrin restores TNF- $\alpha$-mediated downregulation of cyclin A promoter activity and $m R N A$ expression. Since we have previously demonstrated that TNF- $\alpha$ acts by downregulating cyclin A transcription, resulting in EC cell cycle arrest (9), we hypothesized that improvement in EC proliferation by DN ezrin, despite TNF- $\alpha$ exposure, might reflect reduced CHR bonding activity and thus 

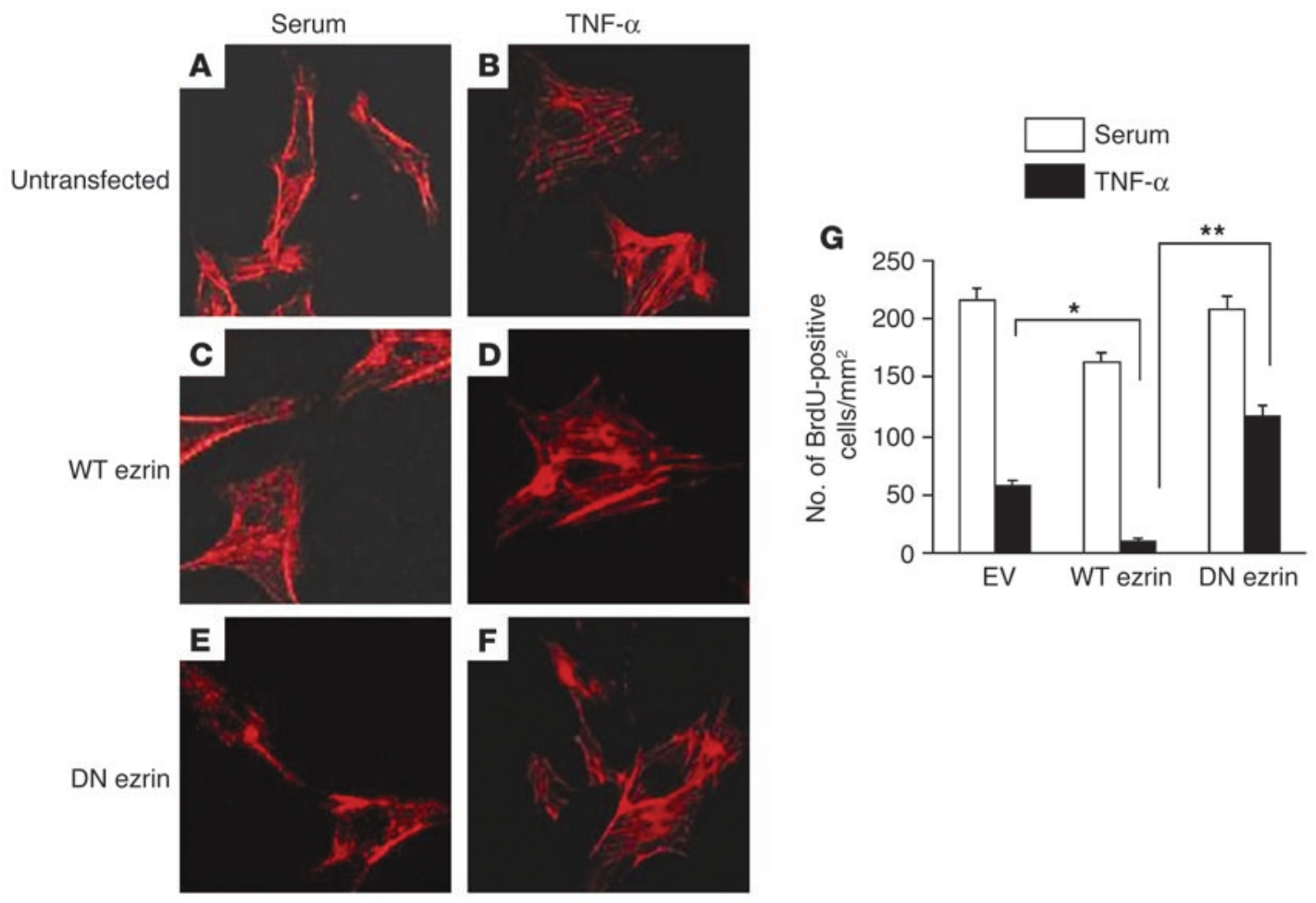

Figure 3

Ezrin mediates TNF- $\alpha$-induced inhibition of EC proliferation. (A-F) Overexpression of WT or DN ezrin does not affect actin cytoskeleton. ECs either untransfected (A) or transfected with WT or DN ezrin (C and E, respectively) and treated (B, D, and F) or not treated with $20 \mathrm{ng} / \mathrm{ml}$ of TNF- $\alpha$ were stained with rhodamine-phalloidin for the detection of actin filaments, using the protocol described elsewhere (59). (G) Quiescent BAECs transiently transfected with empty vector (EV), WT ezrin, or DN ezrin were cultured in serum (white bars) or serum containing $20 \mathrm{ng} / \mathrm{ml}$ of TNF- $\alpha$ (black bars) for 24 hours. Cells were labeled with BrdU $(50 \mu \mathrm{g} / \mathrm{ml})$ for the last 6 hours of culture. Cells were fixed and stained with anti-BrdU antibodies. BrdU-positive cells were counted in at least 6 different visual fields. Average data from 3 similar experiments are shown. ${ }^{*} P<0.01 ;{ }^{* *} P<0.001$.

lead to enhanced cyclin A promoter activity and mRNA expression. We first evaluated the effect of DN ezrin overexpression on cyclin A CHR-binding activity in the nuclear extracts from transiently transfected cells. As shown in Figure 4A, overexpression of DN ezrin markedly abrogated both basal and TNF- $\alpha$-induced upregulation of CHR-binding activity observed in WT ezrin-transfected cells. To examine the effect of TNF- $\alpha$ on cyclin A promoter activity in ezrin expression vector-transfected cells, ECs were cotransfected with WT or DN ezrin and a cyclin A promoter-reporter construct (cycA-luc $-924 /+100$ ), and changes in luciferase activity in comparison with that of cells transfected with control luciferase vector (pGL2) were determined. Promoter activity in empty vector-transfected cells was significantly reduced in TNF- $\alpha$-treated cells $(P<0.01)$ and was further reduced in cells transfected with WT ezrin in both the absence and the presence of TNF- $\alpha$ (Figure 4B). Overexpression of DN ezrin significantly attenuated the negative effect of TNF- $\alpha$ on cyclin A promoter activity compared with that observed in WT ezrin-transfected and empty vector-transfected cells. The effect of DN ezrin on TNF- $\alpha$-mediated cyclin A promoter activity was further corroborated by significant upregulation of cyclin A mRNA expression (Figure 4C). Together these data reveal that blockade of ezrin function abrogates the effect of TNF- $\alpha$ on cyclin A promoter activity, cyclin A expression, and EC proliferation.

TNF- $\alpha$-induced ezrin modulations in ECs are mediated by RhoA kinase signaling. Since ezrin is known to be a target for tyrosine phosphorylation and since activation of ezrin via phosphoryla- tion events is suggested to occur through RhoA signaling in some cell types, we examined the role of RhoA kinase (ROCK-2) in TNF- $\alpha$-induced functional changes in ezrin behavior. First, we determined whether TNF- $\alpha$ treatment of ECs could induce the phosphorylation of ROCK-2. As shown in Figure 5A, treatment of ECs with TNF- $\alpha$ induced ROCK-2 phosphorylation in a time-dependent manner beginning as early as 5 minutes after TNF- $\alpha$ treatment with persistent expression at all time points studied. Cotreatment of cells with the Rho kinase-specific (RhoK-specific) inhibitor Y27632 $(20 \mu \mathrm{M})$ at the time of TNF- $\alpha$ exposure completely abolished ROCK-2 phosphorylation, thereby confirming the specificity for ROCK-2 activation in response to TNF- $\alpha$. TNF- $\alpha$ treatment of ECs also induced timedependent ezrin phosphorylation, which was completely inhibited by Y27632 (Figure 5A). That TNF- $\alpha$-induced activation of ROCK- 2 in turn phosphorylates ezrin was further evident by suppression of ezrin phosphorylation in cells transfected with DN ezrin compared with mock-transfected cells (Figure 5B). To determine whether inhibition of TNF- $\alpha$-induced ROCK-2 activation could abrogate TNF- $\alpha$-mediated upregulation of cyclin A CHR-binding activity, nuclear extracts from ECs, cotreated with TNF- $\alpha$ and Y27632, were analyzed for CHR-binding activity. As indicated in Figure 5C, Y27632 significantly reduced TNF- $\alpha$-induced CHR-binding activity, suggesting that inhibition of ROCK-2 activation blocked ezrin phosphorylation and resultant nuclear translocation. 

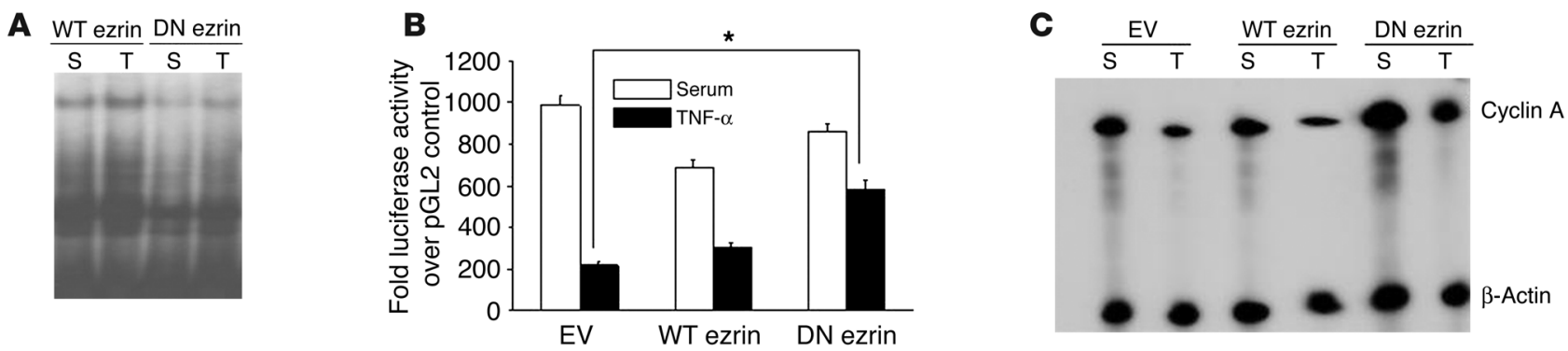

Figure 4

DN ezrin attenuates TNF- $\alpha$ effects on cyclin A. (A) Nuclear extracts from serum-treated and TNF- $\alpha$-treated BAECs, transfected with WT or DN ezrin, were evaluated for CHR-binding activity in EMSAs. Overexpression of DN ezrin significantly blunted TNF- $\alpha$-induced upregulation of $C H R$-binding activity. (B) BAECs cotransfected with cyclin A promoter-reporter construct and either WT or DN ezrin constructs and cultured in the presence or absence of TNF- $\alpha$ for 24 hours were analyzed for luciferase activity. The bar graph represents average promoter activity from 3 similar experiments. ${ }^{*} P<0.01$. (C) Total RNA from empty vector-, WT ezrin-, or DN ezrin-transfected cells under serum or TNF- $\alpha$ treatment conditions was evaluated for cyclin A mRNA expression by ribonuclease protection assays. A representative autoradiograph from 3 similar experiments is shown.

Inbibition of RhoK activation partially attenuates TNF- $\alpha$-inhibitory effects on EC proliferation. RhoK signaling in TNF- $\alpha$-mediated ezrin modulation and resultant EC dysfunction was further confirmed by additional functional experiments. Treatment of ECs with Y27632 partially abrogated the inhibitory effect of TNF- $\alpha$ on EC proliferation, as evident by thymidine uptake assays (Figure 6A). Cotreatment of ECs with Y27632 in the presence of TNF- $\alpha$ had a similar positive effect on cyclin A promoter activity (Figure 6B), as was observed in cells in which DN ezrin was overexpressed. Furthermore, coexpression of dominant-negative RhoK in cells transfected with WT ezrin rescued cells from TNF- $\alpha$-mediated inhibition of proliferation. Conversely, constitutively active RhoK overexpression by itself substantially reduced EC proliferation (Figure 6C).

Transplantation of DN ezrin-transfected human umbilical vein ECs improves blood flow recovery in murine hind limb ischemia. Our prior studies have shown that in vivo blockade of TNF- $\alpha$ function in certain settings leads to increased EC proliferation and function in vivo $(6,7)$. To begin evaluating the effects of ezrin on EC function in vivo, we used the mouse hind limb ischemia model. In this model, previous studies have shown that EC proliferation is crucial to angiogenesis, which results in the formation of collateral vessels and recovery of perfusion of the ischemic tissue (20). Hind limb ischemia was established in nude mice, and human umbilical vein ECs (HUVECs) transfected with WT or DN ezrin and labeled with fluorescent dye 1,1'-dioctadecyl-3,3,3'3'-tetramethylindo-carbocyanine perchlorate (DiI; Invitrogen Corp.) were injected into the ischemic muscle along with the implantation of a BrdU micropump (ALZET; DURECT Corp.). Laser Doppler imaging (Figure 7) revealed that while hind limb perfusion was equally reduced in both groups of animals immediately following surgical excision of the femoral artery (blue denotes decreased blood flow in the operated limbs; blue arrows in Figure 7A), at 7 days after operation the ratio of blood flow in the ischemic versus nonischemic limb was significantly improved in mice receiving DN ezrin-transfected HUVECs compared with those receiving an equal number of transplanted ECs transfected with WT ezrin (red arrow in Figure 7A, black bars in Figure 7B).

To determine the proliferation of transplanted and host ECs, histological sections obtained from mice transplanted with WT ezrin-overexpressing HUVECs (Supplemental Figure 5A) and with DN ezrin-overexpressing HUVECs (Supplemental Figure 5B) were immunostained for BrdU (purple), isolectin B4 (IB4) (green), and
DiI (red). BrdU/DiI-double positive cells in the ischemic hind limb tissues were quantified in at least 8 high visual fields. Data depicted in Figure 8A revealed a significantly higher number of proliferating DN ezrin-transfected HUVECs versus WT ezrin transfectants (pink arrows in Supplemental Figure 5; DN ezrin, $84 \pm 17 / \mathrm{mm}^{2}$; WT ezrin, $\left.28 \pm 13 / \mathrm{mm}^{2} ; P<0.001\right)$. The number of BrdU/IB4-double positive cells (negative for DiI), which denotes endogenous EC proliferation, was similar in tissues from both groups (white arrows in Supplemental Figure 5, and quantified in Figure 8A), indicating that transplantation of HUVECs overexpressing WT or DN ezrin does not modulate endogenous EC proliferation.

To further substantiate this conclusion and to rule out the contribution of secreted factors in response to ezrin transfection, conditioned medium from cultured HUVECs overexpressing either WT or

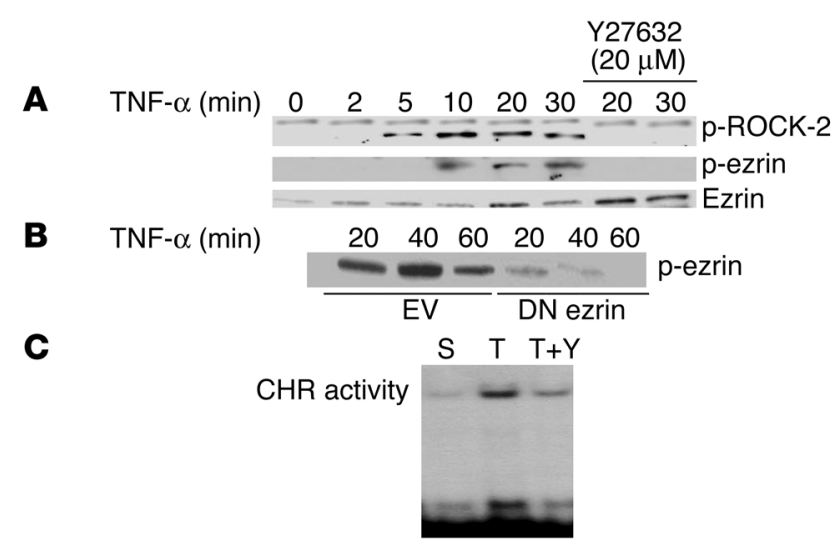

\section{Figure 5}

TNF- $\alpha$-induced activation of ROCK-2 phosphorylates and modifies ezrin function. (A) Total cellular lysates from BAECs treated with TNF- $\alpha$ $(20 \mathrm{ng} / \mathrm{ml})$ for the indicated times in the presence or absence of specific RhoK inhibitor Y27632 (20 $\mu \mathrm{M})$ were analyzed in Western blot for ROCK-2 and phospho-ezrin protein expression. (B) ECs transiently transfected with empty vector or DN ezrin were treated with TNF- $\alpha$ for the indicated times. Total cellular lysates from transfected cells were analyzed for phospho-ezrin. Overexpression of DN ezrin substantially reduced TNF- $\alpha$-induced ezrin phosphorylation. (C) BAEC nuclear extracts treated with serum, TNF- $\alpha$, or TNF- $\alpha$ plus Y27632 were analyzed for $\mathrm{CHR}$-binding activity by EMSA assays. A representative autoradiograph from 3 experiments is shown. 
A

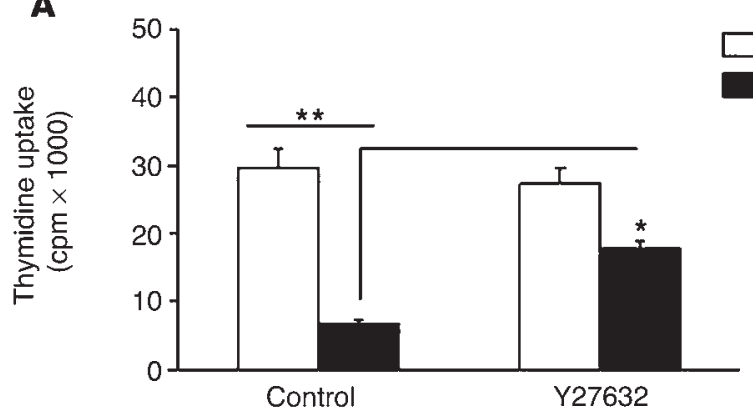

C

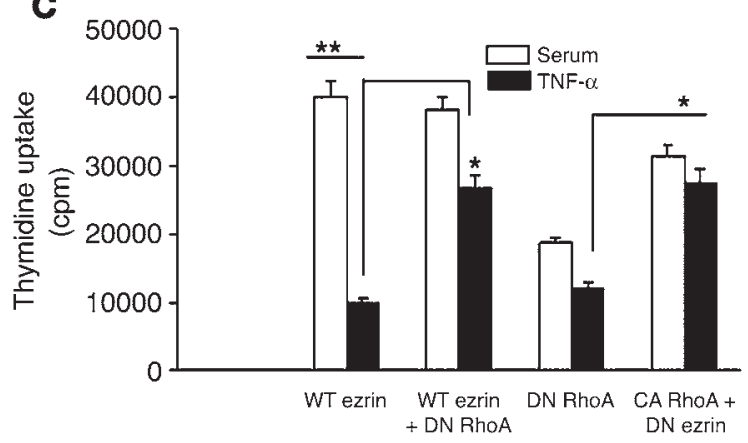

B

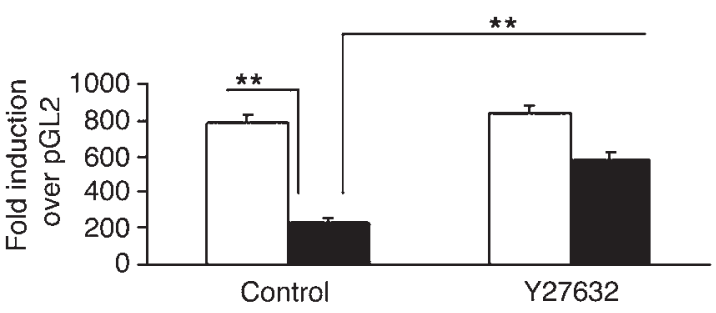

Figure 6

Inhibition of RhoK partially attenuates TNF- $\alpha$-mediated inhibitory effects on EC proliferation and cyclin A promoter activity. (A and B) Cotreatment of cells with Y27632 in the presence of TNF- $\alpha$ attenuates the inhibitory effect of TNF- $\alpha$ as measured by EC proliferation (A) and cyclin A promoter activity (B). (C) Overexpression of constitutively active (CA) RhoK mimics the effect of TNF- $\alpha$ on EC proliferation, while dominantnegative (DN) RhoA reverses it, as measured by ${ }^{3} \mathrm{H}$-thymidine uptake assay. ${ }^{*} P<0.01$; ${ }^{* \star} P<0.001$.

DN ezrin was used in hind limb ischemia models to determine their impact on the recovery of capillary density. Spent medium from WT ezrin- and DN ezrin-overexpressing HUVECs was injected into the ischemic hind limbs of athymic nude mice. Capillaries perfused with FITC-conjugated BS-1 lectin in gastrocnemius muscles were identified by fluorescence confocal microscopy and quantified. Data shown in Figure 8B indicate that although capillary densities in spent medium-injected limbs were significantly higher than in limbs injected with control medium, no difference was observed in capillary numbers between limbs injected with spent medium from either WT ezrin- or DN ezrin-overexpressing HUVECs. These data were also confirmed in vitro (Supplemental Figure 4) by HUVEC proliferation. Conditioned medium from WT ezrin- or DN ezrintransfected cells had no differential effect on HUVEC proliferation (Supplemental Figure 4A). In addition, scratch assays revealed that WT or DN ezrin overexpression also did not affect the motility of the ECs (Supplemental Figure 4B). To determine whether enhanced blood flow recovery in limbs transplanted with DN ezrin-overexpressing HUVECs reflects enhanced capillary density and incorporation of proliferating HUVECs into capillaries, additional experiments were performed in athymic nude mice in which WT ezrin- or DN ezrin-overexpressing DiI-stained HUVECs were transplanted into ischemic hind limbs immediately after the surgery. To specifically measure capillary density and avoid nonspecific staining in the tissue sections, mice were perfused with FITC-conjugated BS-1 lectin. Immediately after perfusion, gastrocnemius muscles were collected, fixed, and sectioned and subjected to laser confocal microscopy to identify fluorescent capillaries and transplanted DiI-labeled HUVECs. Data shown in Figure 8C indicate that capillary densities were significantly higher in the ischemic hind limbs of mice injected with DN ezrin-overexpressing cells. Moreover, a higher number of these cells colocalized with the capillaries, suggesting a "constructive" effect. Taken together, these data suggest that while general enhancement in perfusion of ischemic hind limbs transplanted with ECs expressing WT or DN ezrin could be attributed to paracrine effects of growth factors secreted by HUVECs, the increased perfusion of hind limbs transplanted with DN ezrin-expressing ECs is explained, at least in part, by increased proliferation and incorporation of these cells in the collateral vessels. The precise role of ezrin in neovascularization will, however, require further confirmation in gene knockout animals.

These data reveal that ezrin modulates EC proliferation in vivo, corroborating our in vitro data, and also show that the increase in EC proliferation resulting from inhibition of ezrin function is associated with an increase in physiologic EC function, manifest as a more rapid recovery of hind limb perfusion. These data suggest that under certain in vivo conditions EC function is improved, in this case resulting in enhanced recovery of hind limb perfusion, when ezrin function in impaired.

\section{Discussion}

Under normal conditions in postnatal mammals, ECs lining blood vessel walls are quiescent. Physiologic and pathologic stimuli can induce ECs to enter the cell cycle for the purposes of vascular repair or for the formation of new vessels, e.g., during wound healing, endometrial proliferation, or tumor growth. Accordingly, the regulation of EC proliferation plays a critical role in health and disease. Our data reveal that ezrin plays a pivotal role in the regulation of EC proliferation, acting as a TNF- $\alpha$-inducible transcriptional repressor of cyclin $\mathrm{A}$. 
A
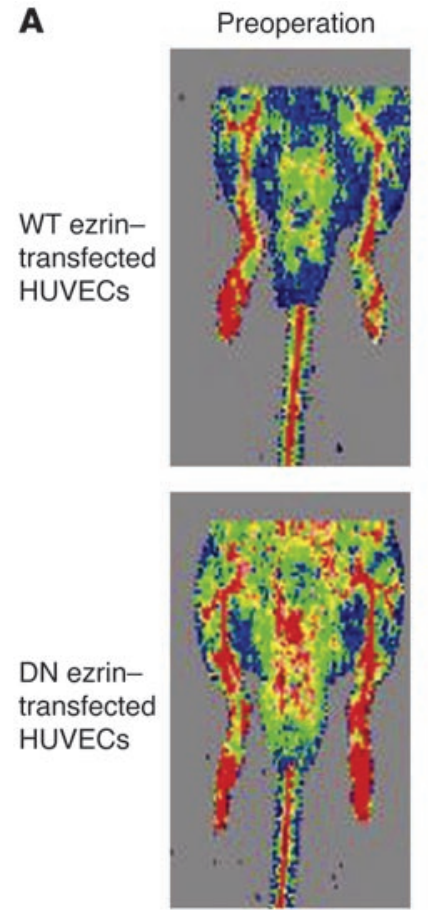

Postoperation
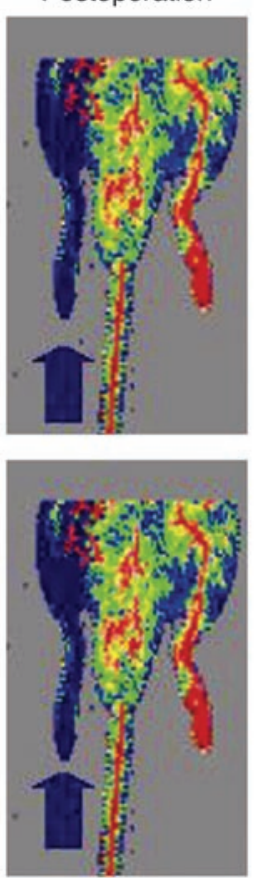

Day 7
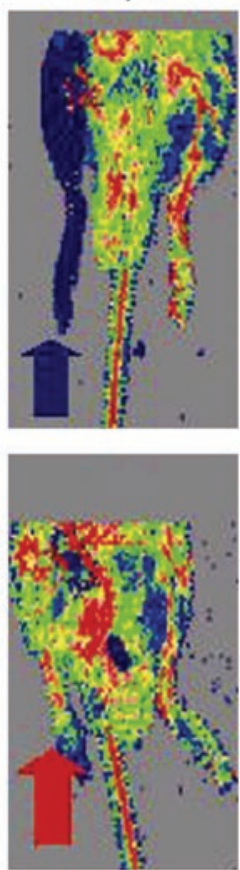

B

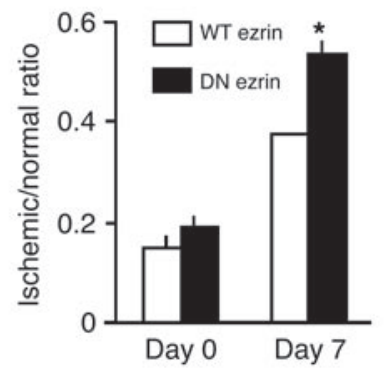

\section{Figure 7}

DN ezrin-transfected HUVECs facilitate angiogenesis in ischemic hind limb. HUVECs transfected with WT or DN ezrin and labeled with Dil were injected into the ischemic muscle. A BrdU micropump was implanted at the time of surgery. (A) Representative pictures of laser Doppler imaging showing that at 7 days after operation the ratio of blood flow in the ischemic (blue arrows) versus nonischemic (red arrow) limb was improved in mice receiving DN ezrin-transfected HUVECs compared with those receiving an equal number of transplanted ECs transfected with WT ezrin. (B) Quantification of postsurgery blood flow in ischemic versus control limbs obtained from 12 mice in each group. ${ }^{*} P<0.02$.

TNF- $\alpha$ is a pleiotropic cytokine that has been shown to influence endothelial proliferation in vitro and to modulate physiologic processes that require EC proliferation in vivo, such as angiogenesis and re-endothelialization after arterial injury. Blockade of TNF- $\alpha$ in vivo, using a soluble receptor molecule, has been shown to accelerate endothelial recovery after arterial injury. On the other hand, null mutations of the TNF- $\alpha$ receptor have been associated with defective angiogenesis in certain settings. These apparently conflicting data reveal that TNF- $\alpha$ 's role in EC physiology is highly context dependent and that a clearer understanding of the mechanisms that underlie TNF- $\alpha$ 's influence on EC proliferation is critical.

In the present study we provide evidence that ezrin, in response to TNF- $\alpha$, mediates cyclin A transcriptional repression in ECs. To the best of our knowledge, this is the first study to demonstrate the function of a cytoskeletal protein in transcriptional regulation of a cell cycle regulatory gene. Several lines of evidence support our conclusion: First, oligonucleotides containing CHR elements from the cyclin A promoter interact specifically with ezrin in nuclear extracts of ECs exposed to TNF- $\alpha$; recombinant ezrin specifically binds to the CHR element, and immunodepletion of ezrin prevents TNF- $\alpha$-mediated upregulation of CHR-binding activity in ECs, which is reconstituted by recombinant ezrin. Second, in response to TNF- $\alpha$, both endogenous and exogenous ezrin translocates to the nucleus; ChIP verifies that ezrin binds to the cyclin A promoter in vivo following TNF- $\alpha$ exposure of ECs. Third, overexpression of DN ezrin attenuates TNF- $\alpha$-induced downregulation of cyclin A promoter activity and mRNA expression, abrogates TNF- $\alpha$-induced repression of EC proliferation in vitro and in vivo, and facilitates angiogenesis in vivo. Finally, our data show that TNF- $\alpha$-induced changes in ezrin localization and function are mediated by the RhoA signaling pathway, since inhibition of RhoK mimics the effects of DN ezrin on CHRbinding activity, cyclin A promoter activity, and EC proliferation. Taken together, these biochemical and functional data identify a novel function of the cytoskeletal protein ezrin in the control of cyclin A gene transcription and EC proliferation and suggest that blockade of TNF- $\alpha$-mediated ezrin modulation in ECs could potentially be used to modulate endothelial growth and function and may have therapeutic implications in conditions that require EC proliferation.

The expression of ERM proteins has been analyzed in various tissues and culture cell lines. Although coexpressed in most cultured cells, they exhibit tissue-specific expression in various organ systems $(21,22)$. On the cellular level, intestinal epithelial cells express only ezrin and hepatocytes express only radixin (23). Although no overt phenotypic changes or defects were observed in moesin and/or radixin knockout mice or in the cells depleted of individual ERM proteins $(16,24)$, which suggests functional redundancy of ERM proteins, the tissue- and cell-specific distribution of ERM proteins argues for some degree of functional dichotomy, which requires closer scrutiny of their functions in a cell-specific manner. Our data, revealing modulation of EC proliferation by manipulation of ezrin alone, provide evidence that this is the case. Modulation of individual ERM protein expression in response to extracellular stimuli has been reported previously $(25,26)$, corroborating our finding of TNF- $\alpha$-induced 
A
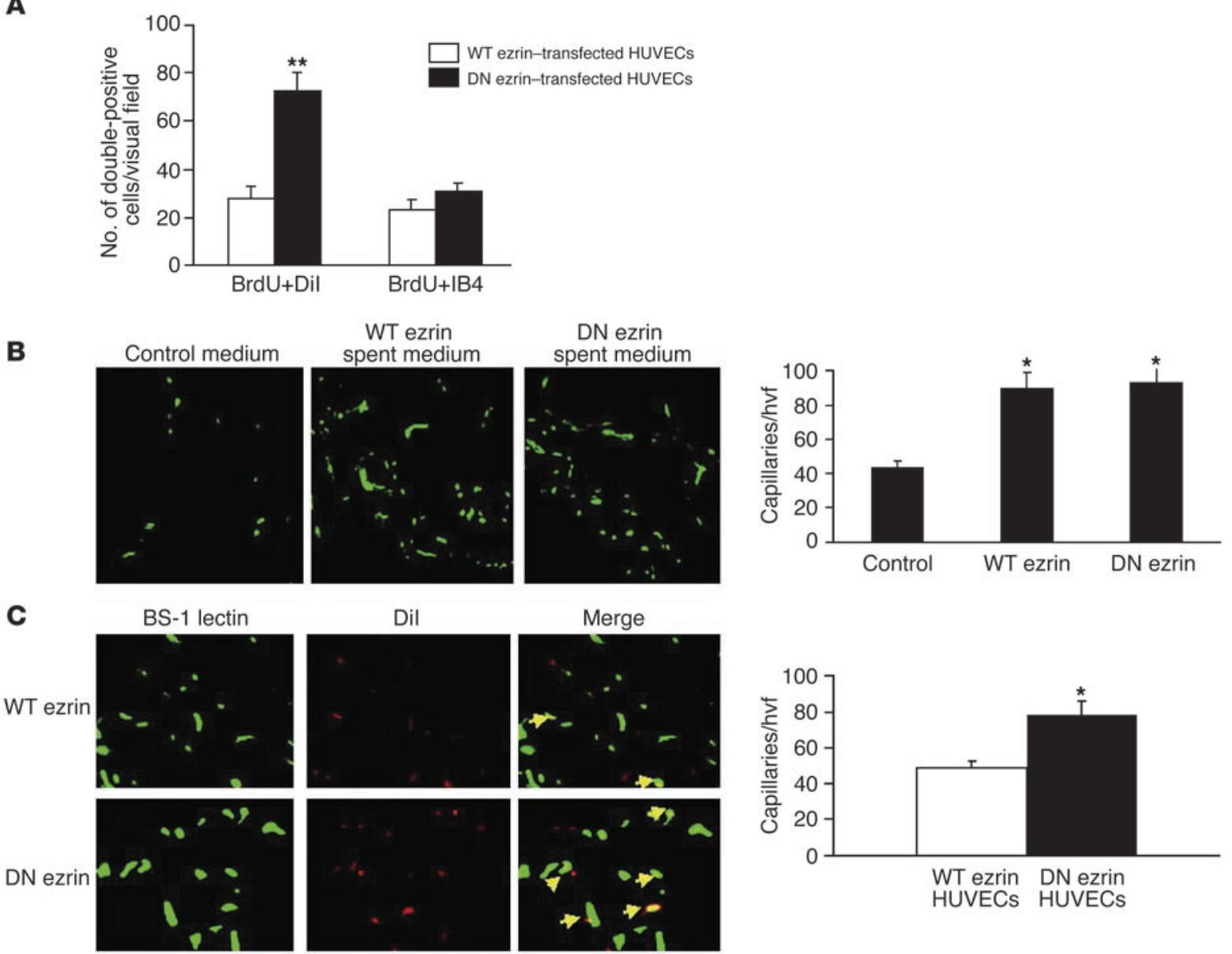

Figure 8

Overexpression of DN ezrin enhances the proliferation of transplanted HUVECs and enhances capillary density. (A) Proliferating ECs in the hind limb tissues were identified by BrdU incorporation and were counted in 8 randomly selected microscope fields from randomly selected sections of tissue from each animal and quantified. (B) Mice receiving control or spent medium after hind limb surgery were perfused with FITC-BS-1 lectin. Fluorescent capillaries were identified by confocal microscopy, counted, and quantified. (C) Tissue sections from indicated animals were subjected to confocal microscopy to visualize BS-1 lectin-perfused capillaries (green) and Dil-labeled HUVECs (red) and quantified. The merge images on the right show capillaries double-positive for BS-1 lectin and Dil (yellow). hvf, high visual field. ${ }^{*} P<0.01 ;{ }^{* *} P<0.001$.

ezrin upregulation in ECs; however, these prior studies did not elucidate the functional impact of these modifications of ezrin revealed in the present studies.

Depending on individual cell types, ERM proteins have been reported to be localized to different structures including microvilli, membrane ruffles, filopodia, uropods, retraction fibers, and cell adhesion sites $(12,22)$. However, this localization of ezrin is controversial $(11,27)$, since ezrin localization has been shown to be cell type specific. The TNF- $\alpha$-induced nuclear localization of ezrin reported in this study, although novel and intriguing, is not without parallel. In normal and transformed human cells, Kaul et al. identified a truncated ezrin isoform in the nucleus (28). Also, growth factor-dependent cytoplasmicto-nuclear translocation of another ERM homolog, Pez, has been reported in ECs (29). Similarly, an alternatively spliced isoform of merlin, a cytoskeletal protein highly homologous to ezrin, was shown to translocate into the nucleus in NIH $3 \mathrm{~T} 3$ cells (30). None of these reports, however, studied the consequences of these nuclear translocations in terms of possible binding of these nuclear proteins to target promoters and regulation of gene transcription. Our data, on the other hand, clearly demonstrate that ezrin translocates to the nucleus of ECs in response to TNF- $\alpha$, binds to the promoter, and represses the transcription of cyclin A and thereby inhibits EC proliferation. It remains a possibility that nuclear translocation of ezrin is dependent on a yet-to-be-identified protein that, in response to TNF- $\alpha$, may bind with ezrin and chaperone it to the nucleus. This hypothesis, however, is unsupported by the present data and remains to be validated in future studies.

Initially, ERM proteins were considered as structural linkers between the plasma membrane and the actin cytoskeleton. Recent studies, however, have implicated ERM proteins as signal transducers involved in a wide variety of cellular functions (16). Ezrin, for example, has been shown to be involved in functions as diverse as cell morphogenesis, adhesion and motility, cellular proliferation, apoptosis, and cell survival $(16,17)$. In a breast carcinoma cell line (MCF-7), ezrin complexes with PKC- $\alpha$ and controls cell motility (31). Ezrin has also been implicated in FasL-mediated 
apoptosis (32-34). On the other hand, ezrin interactions with the $\mathrm{PI} 3 \mathrm{~K} /$ Akt pathway appeared to mediate cell survival signals in a kidney-derived epithelial cell line, LLC-PK1 (35).

There is increasing evidence regarding the role of ezrin in cell proliferation. Most of these studies, involving cancer tissues and cell lines, have reported a direct relationship between cell proliferation and the level of ezrin expression in both malignant $(26,36)$ and nonmalignant cells (37). In RS-4 mouse fibroblasts, microinjection of anti-ezrin antibodies into the cytoplasm blocked their entry into $S$ phase, suggesting a proliferative function of ezrin (38). Our data identify entirely novel functions of ezrin in ECs in vitro and in vivo. In response to TNF- $\alpha$, ezrin inhibits EC proliferation via transcriptional repression of cyclin $\mathrm{A}$, a key cell cycle regulatory protein. In vivo, ezrin is shown to inhibit EC proliferation and thereby inhibits angiogenesis, impairing blood flow recovery in a model of hind limb ischemia. The probable role of TNF- $\alpha$ in ischemia-induced angiogenesis, which is invariably accompanied by recruitment of inflammatory cells, suggests that TNF- $\alpha$-mediated ezrin activation is at play.

This inhibitory effect of ezrin on EC proliferation is in contrast to the studies cited above. It should be noted, however, that several direct and indirect lines of evidence suggest an antiproliferative role for ezrin in various cell systems. In colorectal carcinoma cells, for example, inhibition of ezrin using antisense oligonucleotides increases their motility and invasive behavior, suggesting that ezrin expression in fact inhibits proliferation (39). In a separate study, FGF stimulation induced ezrin expression in rat chondrosarcoma cells, resulting in the induction of growth arrest in the $\mathrm{G}_{1}$ phase (40). Moreover, structurally similar and highly homologous proteins, merlin and DAL-1, have been shown to act as tumor suppressors whose overexpression inhibits cell proliferation (41-43). The rather contrasting roles of ezrin in cell invasion and metastasis on one hand, and antiproliferative effect on the other, probably involve its different roles in cell motility, survival, and proliferation in a cell- and tissue-specific and stimulus-specific manner. It is noteworthy that despite the established role of ezrin and other ERM proteins in the process of cellular growth, proliferation, and apoptosis, no information is available regarding their impact on the regulation of cell cycle and cell cycle regulatory genes. Our data reveal that TNF- $\alpha$-mediated modulation in ezrin expression and distribution negatively regulates cyclin A transcription via binding to transcriptional repressor CHR elements. This study thus identifies an entirely novel function of ezrin in ECs.

We have also shown in this study that the RhoA signaling pathway mediates TNF- $\alpha$-induced changes in ezrin in ECs. TNF- $\alpha$ activates the RhoA downstream kinase RhoK, which in turn phosphorylates ezrin (Figure 5). ERM proteins form intramolecular and intermolecular associations between their amino and carboxyl termini, which cause the proteins to adopt closed conformations in which membrane and cytoskeletal binding sites are masked. Thus, these proteins need to be conformationally activated to fulfill their biological functions. Although TNF- $\alpha$-mediated activation/phosphorylation of ERM proteins has not yet been described, several stimuli such as thrombin, EGF, IGF, and IL-1 $(26,27,40,44)$ are known to activate ERM proteins, leading to the disruption of intramolecular associations and allowing intermolecular associations to occur (17). Activation of ERM proteins including that of ezrin has been shown to involve the induction of various signaling pathways, depending on the stimuli and cell type. Although ezrin activation via the PI3K/Akt pathway and
PKC pathway has been reported, the majority of studies implicate the RhoK pathway in the activation of ezrin $(35,45-47)$. Phosphorylation of ezrin via activation of the RhoA signaling cascade by TNF- $\alpha$ is consistent with the findings of other studies showing RhoA-mediated ERM protein activation (13). However, we did not observe any changes in the morphology of ECs after TNF- $\alpha$ treatment, which suggests that the activated RhoA in ECs may not induce cytoskeletal changes (Figure 3, A-F). This is also consistent with the findings of a prior study in which RhoK-dependent phosphorylation of moesin in neuronal H19-7/IGF-IR cells did not induce any cytoskeletal changes (48). Similarly, in other neuronal cells, Rho signaling has been shown to mediate functions other than cytoskeletal modifications, including axonal outgrowth, dendrogenesis, cell migration, and exo- and endocytosis (49). Taken together, it appears that TNF- $\alpha$-induced RhoA activation and activated-Rho-mediated phosphorylation of ezrin in ECs may affect functions other than the cytoskeletal changes that have been the predominant focus of prior studies.

Restenosis, the recurrent narrowing of arteries after balloon angioplasty or deployment of an intra-arterial stent, remains a significant clinical problem that undermines the benefits of these widely used procedures. Trauma of the vessel during balloon angioplasty triggers a cascade of events including the proliferation and migration of normally quiescent ECs. Optimally, this leads to anatomic and functional endothelial recovery at the sites of vascular injury $(50,51)$, with attendant limitation of neointimal lesion formation. Recent studies have demonstrated that TNF- $\alpha$, expressed locally at the sites of arterial injury, delays re-endothelialization by inhibiting EC cell cycle regulatory genes, such as cyclin A, and enhancing apoptosis, thereby contributing to the development of restenosis $(6,9,52)$. Blockade of TNF- $\alpha$ in vivo using a soluble TNF- $\alpha$ receptor molecule accelerated functional endothelial recovery and inhibited neointimal lesion formation after balloon angioplasty (7). The present studies clarify a previously unknown mechanism by which TNF- $\alpha$ impairs endothelial recovery. Moreover, these studies implicate the RhoK pathway in mediating this effect of TNF- $\alpha$. Recent studies in animal models of atherosclerosis and restenosis have proposed a role for RhoK in the pathophysiology of these diseases. In a porcine model, Morishige et al. (53) have shown that RhoK was functionally upregulated in IL- $\beta$ treated arteriosclerotic coronary lesions in vivo and that selective inhibition of RhoK by gene transfer of its dominant-negative mutant caused a marked regression of the coronary-constrictive remodeling. Very interestingly, RhoK-mediated phosphorylation of ERM proteins was also upregulated within the coronary lesions. Similarly, activation of RhoK and enhanced expression of ezrin were reported after balloon angioplasty in a pig model of femoral artery denudation (54). Inhibition of RhoK in this study significantly reduced neointimal thickening. These findings, although not directly linking ezrin phosphorylation as the underlying mechanism for positive functional outcome, are suggestive of this connection. Also, it is not clear that inhibition of neointima formation by dominant-negative RhoK depicts an inhibitory effect on VSMCs or a proliferative effect on ECs and consequent re-endothelialization of denuded artery. Further studies on specific effects of RhoK and ezrin phosphorylation on these 2 cell types within the vessel would help clarify these issues.

In sum, the role of ezrin in regulating EC proliferation provides a novel therapeutic target that could be exploited to modulate therapeutic or pathologic angiogenesis and that may provide clues 
to the advent of conditions, such as atherosclerosis, in which the integrity of the endothelium, which is thought to be maintained by occasional bursts of EC proliferation, fails.

\section{Methods}

Cell culture. BAECs and HUVECs were isolated and cultured as previously described $(6,9)$.

Recombinant protein, constructs, transient transfections, and reporter assays. Recombinant human ezrin protein (GST-ezrin) and WT ezrin (pCB6ezrin-VSVG) and DN ezrin (pCB6-ezrin-Nter-VSVG) expression vectors were a kind gift from M. Arpin (Morphogenese et Signalisation Cellulaires, CNRS-Institut Curie, Paris, France) (55). Constitutively active RhoK (V14) and dominant-negative RhoK (C3) were a kind gift from R.K. Assoian (University of Pennsylvania School of Medicine, Philadelphia, Pennsylvania, USA). The cyclin A promoter-luciferase reporter construct (cycA-Luc $-924 /+100$ ) has been described before (9). For cyclin A promoter activity, BAECs were transiently transfected with cyclin A promoter-luciferase reporter construct $(-924 /+100)$ using TransFast (Promega) according to the manufacturer's instructions. Cells were trypsinized, pooled, and replated according to experimental requirements and were treated or not treated with $20 \mathrm{ng} / \mathrm{ml}$ recombinant human TNF- $\alpha$ for 24 hours. Cells were harvested and assayed for luciferase activity using a Berthold Technologies Lumat LB 9501 luminometer. Luciferase activity was normalized to the alkaline phosphatase activity produced by a cotransfected alkaline phosphatase plasmid (pSVAPAP), which served as transfection-efficiency control. Ezrin expression vectors were similarly transfected. Transfection efficiency was monitored by immunostaining and Western blot analysis of tagged VSVG protein and was comparable among transfections.

Antibodies. Anti-ezrin polyclonal antibodies recognizing 81-kDa ezrin protein were purchased from Upstate. Anti-phospho-ezrin was purchased from Santa Cruz Biotechnology Inc. Anti-VSVG mAbs were from Roche Diagnostics Corp. Santa Cruz Biotechnology Inc. was the source of anti-ROCK-2 antibodies. FITC-anti-IB4 and anti-BrdU antibodies were purchased from Vector Laboratories and BIODESIGN International, respectively.

RNA isolation and ribonuclease protection assay. Synchronized BAECs were released from serum starvation, transfected with either empty vector or ezrin expression vectors, and further cultured in the presence or absence of TNF- $\alpha(20 \mathrm{ng} / \mathrm{ml})$ for the indicated times. Total cellular RNA was isolated using TRIzol reagent (Invitrogen Corp.). Human cyclin multiprobe DNA template (BD Biosciences - Pharmingen) and ( $\left.\alpha^{32} \mathrm{P}\right) \mathrm{UTP}$ (PerkinElmer) were used to synthesize in vitro-transcribed antisense riboprobe, and ribonuclease protection assays (RPAs) were carried out using an RPA III kit (Ambion Inc.) according to the manufacturer's instructions. Briefly, 5-10 $\mu \mathrm{g}$ of RNA was hybridized with radiolabeled probe and was then digested with RNase A/T1. RNase-protected transcripts were then precipitated and were run on $5 \%$ sequencing gels, dried, and autoradiographed.

Oligonucleotides, nuclear extracts, and EMSA. The following oligonucleotides representing CHR elements from the cyclin A promoter (nucleotide -31/-16) were synthesized (MWG Biotech Inc.) and used in EMSA: WT CHR, 5'ATACTTGAACTGCA-3'; and mutant CHR, 5'-ATACTgtccCTGCA-3'. Mutant CHR represents oligonucleotides wherein italicized nucleotides were substituted. Nuclear protein isolation and EMSAs were carried out as described elsewhere (9).

Thymidine uptake assay. Cell proliferation was assayed by thymidine uptake assay as described previously (56).

Southwestern analysis and Western blots. For modified Southwestern assays, nuclear proteins $(50 \mu \mathrm{g})$ were reacted with biotin-labeled WT and mutant CHR oligonucleotides in a scaled-up ( $\times 5$ reaction volumes) EMSA reaction. Biotin-CHR-protein complexes were captured on UltraLink streptavidinagarose beads (Amersham Pharmacia Biotech Inc.). Beads were washed, and eluted proteins were resolved by $10 \%$ SDS-PAGE gels, electrotransferred to PVDF membrane, and rocked for 10 minutes at $4^{\circ} \mathrm{C}$ in binding buffer (25 mM HEPES/KOH, $60 \mathrm{mM} \mathrm{KCl}, 1 \mathrm{mM}$ DTT, $1 \mathrm{mM}$ EDTA, and $6 \mathrm{M}$ guanidinium chloride). The membrane was transferred to binding buffer containing $3 \mathrm{M}$ guanidinium chloride and incubated as in the previous step. Renaturation was achieved by 8 successive washes in binding buffer, each with a $50 \%$ stepwise reduction of guanidinium chloride, and a final 1-hour incubation in the absence of guanidinium chloride plus $5 \%$ nonfat dry milk. The membrane was then incubated with anti-ezrin antibodies (Upstate) in binding buffer plus $0.25 \%$ nonfat dry milk for 2-4 hours at room temperature. Membranes were washed, air-dried, and autoradiographed. Western blots for ezrin, VSVG, phospho-ezrin, and RhoK were done using specific antibodies and standard protocols.

Immunocytochemical staining and laser confocal microscopy. For immunostaining, appropriately transfected and/or treated cells were fixed and endogenous peroxidase activity was blocked. Cells were then incubated with primary antibodies at $37^{\circ} \mathrm{C}$ for 1 hour or at $4^{\circ} \mathrm{C}$ overnight. Primary immune complex was detected with species-matched biotinylated secondary antibodies and streptavidin peroxidase $(7,57)$. For BrdU immunostaining, fixed cells/sections were first incubated in $2 \mathrm{M}$ $\mathrm{HCl}$ for 10 minutes at $37^{\circ} \mathrm{C}$ to denature DNA; this was followed by blocking and primary antibody incubation steps. The slides were mounted with glycerol gelatin aqueous mounting medium and examined on an Olympus Vanox-T microscope (Olympus American Inc.). Pictures were recorded on Kodak GOLD Plus films (Eastman Kodak Co.). Immunofluorescent staining for ezrin translocation was evaluated by laser confocal microscopy as described elsewhere (57).

ChIP. ChIP assays were carried out using a ChIP assay kit from Upstate, following the manufacturer's instructions. Briefly, ECs $\left(3 \times 10^{7}\right.$ per treatment group) were cross-linked by $1 \%$ formaldehyde at room temperature $\left(22^{\circ} \mathrm{C}\right)$ and lysed by sonication in $1 \mathrm{ml}$ of ChIP buffer $(0.1 \%$ SDS, $1 \%$ Triton $\mathrm{X}-100,2 \mathrm{mM}$ EDTA, $150 \mathrm{mM} \mathrm{NaCl}, 20 \mathrm{mM}$ Tris-HCl, pH 8). Chromatin DNA was isolated from the lysates at this stage using gradient centrifugation. Total chromatin was digested using Sau3A1 enzyme before the IP step. Cross-linked DNA and proteins were purified from free un-crosslinked proteins by centrifugation through a gradient of 5-8 $\mathrm{M}$ urea prepared in $10 \mathrm{mM}$ Tris, $\mathrm{pH}$ 8.0, $1 \mathrm{mM}$ EDTA. Chromatin 0.6-1 kb long was washed twice in a buffer of $10 \mathrm{mM}$ Tris, $\mathrm{pH}$ 8.0, $1 \mathrm{mM}$ EDTA, and purified by dialysis. Chromatin was then precleared with $100 \mu \mathrm{g}$ of salmon sperm DNA and $450 \mu \mathrm{l}$ of protein A or protein A plus agarose (Santa Cruz Biotechnology Inc.) at $4^{\circ} \mathrm{C}$ for 30 minutes and then immunoprecipitated with antibodies specific for ezrin or a control antibody at $4^{\circ} \mathrm{C}$ for 5 hours. The precipitates were washed with ice-cold ChIP buffer and washing buffer (0.25 M LiCl, 1\% NP-40, 1\% deoxycholate, 1 mM EDTA, 10 mM Tris-HCl, $\mathrm{pH} 8$ ). The DNA was extracted from the precipitates by incubation with $100 \mu \mathrm{l}$ of TE buffer (10 mM Tris- $\mathrm{HCl}, \mathrm{pH} 8.0$, and $1 \mathrm{mM}$ EDTA), $2.5 \mu \mathrm{l}$ of $10 \% \mathrm{SDS}$, and $5 \mu \mathrm{g}$ of proteinase $\mathrm{K}$ at $45^{\circ} \mathrm{C}$ overnight. After 1 hour of incubation at $65^{\circ} \mathrm{C}$ with an additional $2.5 \mu \mathrm{l}$ of $5 \mathrm{M} \mathrm{NaCl}$, the DNA fragments were purified with a QIAEX II gel purification kit (QIAGEN) and subjected to PCR analysis using primers to amplify the cyclin A promoter encompassing CHR elements. The PCR products were separated on $0.7-1 \%$ agarose gels and visualized by ethidium bromide staining.

Hind limb ischemia, cell transplantation, and laser Doppler imaging. Hind limb ischemia was established in athymic nude mice (Harlan) (58), and HUVECs transfected with WT or DN ezrin and labeled with DiI were injected into the ischemic muscle. A BrdU micropump (ALZET; DURECT Corp.) was implanted at the time of surgery. Laser Doppler imaging to determine blood flow was performed immediately after surgery (day 0 ) and at day 7 after cell injections. Seven days after cell transplantation, the tissues were harvested and assayed by histochemical staining for IB4, BrdU, and DiI followed by 
confocal microscopy. In some experiments, animals were perfused with FITC-BS-1 lectin to identify capillaries before sacrifice and tissue retrieval. All protocols for animal studies were approved by the Institutional Animal Care and Use Committee (IACUC) at St. Elizabeth's Medical Center.

Statistical analyses. All experiments were carried out at least 3 times with similar results. Results are presented as mean \pm SEM. Comparisons were done by ANOVA (GB-STAT; Dynamic Microsystems Inc.) or $\chi^{2}$ test for percentages. All tests were 2 -sided, and a $P$ value less than 0.05 was considered statistically significant.

\section{Acknowledgments}

This work was supported by NIH grants R01 HL63414, HL53354, HL63695, HL66957, and HL57516 (to D.W. Losordo). We thank
M. Arpin for recombinant-ezrin protein and expression vectors, R.K. Assoian for RhoK constructs, and Mickey Neely and Deirdre Costello for excellent secretarial help.

Received for publication July 28, 2004, and accepted in revised form May 10, 2005.

Address correspondence to: Douglas W. Losordo or Raj Kishore, St. Elizabeth's Medical Center, 736 Cambridge Street, Boston, Massachusetts 02135, USA. Phone: (617) 789-3474; Fax: (617) 789-6362; E-mail: douglas.losordo@tufts.edu (D. Losordo). Phone: (617) 562-7547; Fax: (617) 562-7506; E-mail: raj.kishore@ tufts.edu (R. Kishore).
1. Ausprunk, D.H., and Folkman, J. 1977. Migration and proliferation of endothelial cells in preformed and newly formed blood vessels during tumor angiogenesis. Microvasc. Res. 14:53-65.

2. Winkles, J.A., et al. 1987. Human vascular smooth muscle cells both express and respond to heparinbinding growth factor I (endothelial cell growth factor). Proc. Natl. Acad. Sci. U. S. A. 84:7124-7128.

3. Modur, V., Zimmerman, G.A., Prescott, S.M., and McIntyre, T.M. 1996. Endothelial cell inflammatory responses to tumor necrosis factor alpha. Ceramide-dependent and -independent mitogenactivated protein kinase cascades. J. Biol. Chem 271:13094-13102.

4. Leibovich, S.J., et al. 1987. Macrophage-induced angiogenesis is mediated by tumour necrosis factor-alpha. Nature. 329:630-632.

5. Lopez-Marure, R., Bernal, A.E., and Zentella, A. 1997. Interference with c-myc expression and RB phosphorylation during TNF-mediated growth arrest in human endothelial cells. Biochem. Biophys. Res. Commun. 236:819-824.

6. Spyridopoulos, I., et al. 1998. Restoration of E2F expression rescues vascular endothelial cells from tumor necrosis factor-alpha-induced apoptosis. Circulation. 98:2883-2890.

7. Krasinski, K., Spyridopoulos, I., Kearney, M., and Losordo, D.W. 2001. In vivo blockade of tumor necrosis factor-alpha accelerates functional endothelial recovery after balloon angioplasty. Circulation. 104:1754-1756.

8. Lopez-Marure, R., Ventura, J.L., Sanchez, L., Montano, L.F., and Zentella, A. 2000. Ceramide mimics tumour necrosis factor-alpha in the induction of cell cycle arrest in endothelial cells. Induction of the tumour suppressor $\mathrm{p} 53$ with decrease in retinoblastoma/protein levels. Eur. J. Biochem. 267:4325-4333.

9. Kishore, R., Spyridopoulos, I., Luedemann, C., and Losordo, D.W. 2002. Functionally novel tumor necrosis factor-alpha-modulated CHR-binding protein mediates cyclin A transcriptional repression in vascular endothelial cells. Circ. Res. 91:307-314.

10. Vaheri, A., et al. 1997. The ezrin protein family: membrane-cytoskeleton interactions and disease associations [review]. Curr. Opin. Cell Biol. 9:659-666.

11. Bretscher, A., Reczek, D., and Berryman, M. 1997. Ezrin: a protein requiring conformational activation to link microfilaments to the plasma membrane in the assembly of cell surface structures. J. Cell Sci. 110:3011-3018.

12. Tsukita, S., and Yonemura, S. 1999. Cortical actin organization: lessons from ERM (ezrin/radixin/ moesin) proteins. J. Biol. Chem. 274:34507-34510.

13. Matsui, T., Yonemura, S., and Tsukita, S. 1999. Activation of ERM proteins in vivo by Rho involves phosphatidyl-inositol 4-phosphate 5-kinase and not ROCK kinases. Curr. Biol. 9:1259-1262.

14. Gary, R., and Bretscher, A. 1995. Ezrin self-association involves binding of an $\mathrm{N}$-terminal domain to a normally masked C-terminal domain that includes the F-actin binding site. Mol. Biol. Cell. 6:1061-1075.

15. Paglini, G., Kunda, P., Quiroga, S., Kosik, K., and Caceres, A. 1998. Suppression of radixin and moesin alters growth cone morphology, motility, and process formation in primary cultured neurons. J. Cell Biol. 143:443-455.

16. Louvet-Vallee, S. 2000. ERM proteins: from cellular architecture to cell signaling. Biol. Cell. 92:305-316.

17. Gautreau, A., Louvard, D., and Arpin, M. 2002. ERM proteins and NF2 tumor suppressor: the Yin and Yang of cortical actin organization and cell growth signaling [review]. Curr. Opin. Cell Biol. 14:104-109.

18. Wojciak-Stothard, B., Entwistle, A., Garg, R., and Ridley, A.J. 1998. Regulation of TNF-alpha-induced reorganization of the actin cytoskeleton and cellcell junctions by Rho, Rac, and Cdc42 in human endothelial cells. J. Cell. Physiol. 176:150-165.

19. Petrache, I., et al. 2003. Caspase-dependent cleavage of myosin light chain kinase (MLCK) is involved in TNF-alpha-mediated bovine pulmonary endothelial cell apoptosis. FASEB J. 17:407-416.

20. Couffinhal, T., et al. 1998. Mouse model of angiogenesis. Am. J. Pathol. 152:1667-1679.

21. Sato, N., Funayama, N., Nagafuchi, A., Yonemura, S., and Tsukita, S. 1992. A gene family consisting of ezrin, radixin and moesin. Its specific localization at actin filament/plasma membrane association sites. J. Cell Sci. 103:131-143.

22. Berryman, M., Franck, Z., and Bretscher, A. 1993. Ezrin is concentrated in the apical microvilli of a wide variety of epithelial cells whereas moesin is found primarily in endothelial cells. J. Cell Sci. 105:1025-1043.

23. Amieva, M.R., Wilgenbus, K.K., and Furthmayr, H. 1994. Radixin is a component of hepatocyte microvilli in situ. Exp. Cell Res. 210:140-144.

24. Tsukita, S., Oishi, K., Sato, N., Sagara, J., and Kawai, A. 1994. ERM family members as molecular linkers between the cell surface glycoprotein CD44 and actin-based cytoskeletons. J. Cell Biol. 126:391-401.

25. Jiang, W.G., and Hiscox, S. 1996. Cytokine regulation of ezrin expression in the human colon cancer cell line HT29. Anticancer Res. 16:861-865.

26. Chen, Z., et al. 2001. Ovarian epithelial carcinoma tyrosine phosphorylation, cell proliferation, and ezrin translocation are stimulated by interleukin 1alpha and epidermal growth factor. Cancer. 92:3068-3075.

27. Bretscher, A. 2000. The cytoskeleton: from regulation to function. Conference: the 15th Meeting of the European Cytoskeleton Forum. EMBO Rep. 1:473-476.

28. Kaul, S.C., et al. 1999. Identification of a 55-kDa ezrin-related protein that induces cytoskeletal changes and localizes to the nucleolus. Exp. Cell Res. 250:51-61.

29. Wadham, C., Gamble, J.R., Vadas, M.A., and Khew-
Goodall, Y. 2000. Translocation of protein tyrosine phosphatase Pez/PTPD2/PTP36 to the nucleus is associated with induction of cell proliferation. J. Cell Sci. 113:3117-3123.

30. Schmucker, B., Tang, Y., and Kressel, M. 1999. Novel alternatively spliced isoforms of the neurofibromatosis type 2 tumor suppressor are targeted to the nucleus and cytoplasmic granules. Hum. Mol. Genet. 8:1561-1570.

31. $\mathrm{Ng}$, T., et al. 2001. Ezrin is a downstream effector of trafficking PKC-integrin complexes involved in the control of cell motility. EMBOJ. 20:2723-2741.

32. Kondo, T., et al. 1997. ERM (ezrin/radixin/moesin)-based molecular mechanism of microvillar breakdown at an early stage of apoptosis. J. Cell Biol. 139:749-758.

33. Parlato, S., et al. 2000. CD95 (APO-1/Fas) linkage to the actin cytoskeleton through ezrin in human $\mathrm{T}$ lymphocytes: a novel regulatory mechanism of the CD95 apoptotic pathway. EMBOJ. 19:5123-5134.

34. Wang, K.C., Cheng, A.L., Chuang, S.E., Hsu, H.C., and Su, I.J. 2000. Retinoic acid-induced apoptotic pathway in T-cell lymphoma: identification of four groups of genes with differential biological functions. Exp. Hematol. 28:1441-1450.

35. Gautreau, A., Poullet, P., Louvard, D., and Arpin, M. 1999. Ezrin, a plasma membrane-microfilament linker, signals cell survival through the phosphatidylinositol 3-kinase/Akt pathway. Proc. Natl. Acad. Sci. U. S. A. 96:7300-7305.

36. Ohtani, K., et al. 2002. Ezrin, a membrane-cytoskeletal linking protein, is highly expressed in atypical endometrial hyperplasia and uterine endometrioid adenocarcinoma. Cancer Lett. 179:79-86.

37. Crepaldi, T., Gautreau, A., Comoglio, P.M., Louvard, D., and Arpin, M. 1997. Ezrin is an effector of hepatocyte growth factor-mediated migration and morphogenesis in epithelial cells. J. Cell Biol. 138:423-434.

38. Kaul, S.C., Mitsui, Y., Komatsu, Y., Reddel, R.R., and Wadhwa, R. 1996. A highly expressed $81 \mathrm{kDa}$ protein in immortalized mouse fibroblast: its proliferative function and identity with ezrin. Oncogene. 13:1231-1237.

39. Hiscox, S., and Jiang, W.G. 1999. Ezrin regulates cellcell and cell-matrix adhesion, a possible role with E-cadherin/beta-catenin. J. Cell Sci. 112:3081-3090.

40. Rozenblatt-Rosen, O., et al. 2002. Induction of chondrocyte growth arrest by FGF: transcriptional and cytoskeletal alterations. J. Cell Sci. 115:553-562.

41. Gutmann, D.H., et al. 1999. Increased expression of the NF2 tumor suppressor gene product, merlin, impairs cell motility, adhesion and spreading. Hum. Mol. Genet. 8:267-275.

42. Gutmann, D.H., Hirbe, A.C., Huang, Z.Y., and Haipek, C.A. 2001. The protein 4.1 tumor suppressor, DAL-1, impairs cell motility, but regulates proliferation in a cell-type-specific fashion. Neurobiol. Dis. 8:266-278.

43. Morrison, H., et al. 2001. The NF2 tumor suppressor gene product, merlin, mediates contact inhibition 
of growth through interactions with CD44. Genes Dev. 15:968-980.

44. Nakamura, F., Amieva, M.R., and Furthmayr, H. 1995. Phosphorylation of threonine 558 in the carboxyl-terminal actin-binding domain of moesin by thrombin activation of human platelets. J. Biol. Chem. 270:31377-31385.

45. Perez, O.D., et al. 2002. Activation of the PKB/AKT pathway by ICAM-2. Immunity. 16:51-65.

46. Tran, Y.K., et al. 1999. A novel member of the NF2/ ERM/4.1 superfamily with growth suppressing properties in lung cancer. Cancer Res. 59:35-43.

47. Shaw, R.J., Henry, M., Solomon, F., and Jacks, T. 1998. RhoA-dependent phosphorylation and relocalization of ERM proteins into apical membrane/actin protrusions in fibroblasts. Mol. Biol. Cell. 9:403-419.

48. Jeon, S., et al. 2002. RhoA and Rho kinase-dependent phosphorylation of moesin at Thr-558 in hippocampal neuronal cells by glutamate. J. Biol. Chem. 277:16576-16584.

49. Van Aelst, L., and D'Souza-Schorey, C. 1997.
Rho GTPases and signaling networks. Genes Dev. 11:2295-2322.

50. Folkman, J., and Shing, Y. 1992. Angiogenesis. J. Biol. Chem. 267:10931-10934.

51. Lindner, V., and Reidy, M.A. 1996. Expression of VEGF receptors in arteries after endothelial injury and lack of increased endothelial regrowth in response to VEGF. Arterioscler. Thromb. Vasc. Biol. 16:1399-1405.

52. Clausell, N., et al. 1995. Expression of tumor necrosis factor and accumulation of fibronectin in coronary artery restenosis lesions retrieved by atherectomy. Br. Heart J. 73:534-539.

53. Morishige, K., et al. 2001. Adenovirus-mediated transfer of dominant-negative rho-kinase induces a regression of coronary arteriosclerosis in pigs in vivo. Arterioscler. Thromb. Vasc. Biol. 21:548-554

54. Eto, Y., et al. 2000. Gene transfer of dominant negative Rho kinase suppresses neointimal formation after balloon injury in pigs. Am. J. Physiol. Heart Circ. Physiol. 278:H1744-H1750.

55. Algrain, M., Turunen, O., Vaheri, A., Louvard, D., and Arpin, M. 1993. Ezrin contains cytoskeleton and membrane binding domains accounting for its proposed role as a membrane-cytoskeletal linker. J. Cell Biol. 120:129-139.

56. McKallip, R., Li, R., and Ladisch, S. 1999. Tumor gangliosides inhibit the tumor-specific immune response. J. Immunol. 163:3718-3726.

57. Goukassian, D.A., et al. 2003. Engineering the response to vascular injury: divergent effects of deregulated E2F1 expression on vascular smooth muscle cells and endothelial cells result in endothelial recovery and inhibition of neointimal growth. Circ. Res. 93:162-169.

58. Yamaguchi, J., et al. 2003. Stromal cell-derived factor-1 effects on ex vivo expanded endothelial progenitor cell recruitment for ischemic neovascularization. Circulation. 107:1322-1328.

59. Takaishi, K., Matozaki, T., Nakano, K., and Takai, Y. 2000. Multiple downstream signalling pathways from ROCK, a target molecule of Rho small G protein, in reorganization of the actin cytoskeleton in Madin-Darby canine kidney cells. Genes Cells. 5:929-936. 